\title{
Simulation and experimental study on vibration and sound radiation control with piezoelectric actuators
}

\author{
Zhiyi Zhang*, Yong Chen, Hongguang Li and Hongxing Hua \\ State Key Laboratory of Mechanical System and Vibration, Shanghai Jiaotong University, Shanghai, 200240, P.R. \\ China
}

Received 4 February 2010

Revised 11 April 2010

\begin{abstract}
FEM/BEM is adopted to model the interaction between the fluid and structures. In the modeling, modal truncation and inertial coupling are applied to sufficiently reduce the coupled model order. This approach is adopted for the purpose of constructing a modal model in the time domain. Active vibration control is realized with piezoelectric actuators and an adaptive method. In the control, the summation of vibration responses is used as the control error since the integral of acceleration on the plate surface is approximately proportional to the far field sound pressure. A rigidly baffled plate connected with a mass through one piezoelectric actuator is simulated at first. In the experiment, the plate is excited by a rotating eccentric mass and controlled with four piezoelectric actuators. The results have shown that active vibration control with the piezoelectric actuators can lead to a noticeable attenuation in sound pressure.
\end{abstract}

Keywords: Fluid-structure interaction, piezoelectric actuator, adaptive vibration control, sound radiation

\section{Introduction}

Structural vibration and sound radiation have been investigated extensively. Besides analytical methods, numerical ones, such as FEM and FEM/BEM, are powerful and used frequently in the analysis of fluid-structure interaction (FSI) [1-4]. In the computation of FSI, FEM/BEM is usually a preferable method due to its advantage in reducing the degrees of freedom of the coupled systems. For the analysis of steady-state vibration and/or sound radiation, FSI is often treated in the frequency domain. However, the transient fluid-structure interaction, such as structural responses to loads of short time duration, has to be described with time domain methods [5]. In order to simulate active control of vibration and the related structural sound radiation in the time domain, a lower order model with an accurate description of dynamics in the low-medium frequency range is then necessary to numerical simulation. The modeling approach in this study is based on an early work of the authors, adopting FEM/BEM and modal truncation to derive a reduced time domain model [6]. The research on active control of structural sound radiation started in the late 1980s [7-9], and the extensive investigation is mainly attributed to the development of technologies of actuation, sensing and computation. However, compared with the researches about sound radiation in air, there is little work about underwater sound radiation control [10-12], and especially scarce work that deals with active vibration isolation and underwater sound radiation.

Passive control methods are popular in the engineering of vibration control, but in many applications, active control methods have to be adopted to achieve demanded reduction in vibration level at lower frequencies where the passive

\footnotetext{
*Corresponding author. E-mail: chychang@ sjtu.edu.cn.
} 


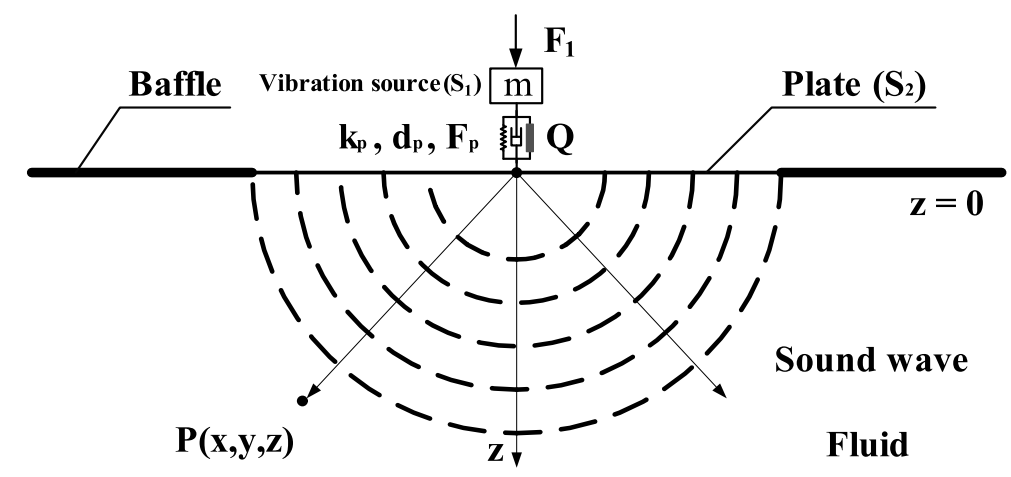

Fig. 1. Rigidly baffled finite plate and active isolator.

control ways are usually not qualified. In the implementation of active vibration control, actuators are of fundamental importance because they determine the realization and complexity of system structures, control laws as well as the performance. Currently, the commonly used actuators in vibration control are electrohydraulic/pneumatic actuators electromagnetic, actuators and smart material actuators, etc., among which the piezoelectric actuators are of attracting features, such as large output force, high efficiency and simple structure, and applied in nano-positioning, high precision manufacturing, vibration and sound radiation control, etc. [13-16]. Polyvinylidenefluoride (PVDF) and lead zirconate titanate (PZT) actuators have been used in practice for vibration control and precise positioning [1719]. Usually, PZT/PVDF actuators are bonded to the surfaces of or imbedded into structures so that structures can response smartly to external disturbance [20-22]. For example, active constrained layer damping (ACLD) treatments can increase structural damping and accordingly reduce structural vibration and sound radiation. For vibration transmission control, different types of piezoelectric actuators have been explored to improve the performance of actuation, and especially the maximum actuation stroke. So far, several models of piezoelectric actuators have been presented and verified [23,24], which forms a sound base for the implementation of piezoelectric actuators in active vibration control.

This paper gives an investigation on the cancellation of vibrations of elastic structures and the induced underwater sound pressure by using piezoelectric actuators. Four sections are organized in the following discussion. First of all, equations of FSI are established for a fluid-plate interaction system in Section 2. A reduced modal model of the elastic plate obtained by finite element analysis is synthesized with the boundary element model of the fluid. Moreover, in this section, an adaptive method for the control of vibration and sound radiation is given. In Section 3 , numerical simulation on the adaptive vibration control and its role in reducing the plate-induced sound pressure is presented. An experimental system and the results on active control of vibration and sound radiation are presented in Section 4. Finally, concluding remarks are given in Section 5.

\section{Mathematical description}

\subsection{Coupled vibration}

Consider the fluid-structure coupled system shown in Fig. 1. As illustrated, $S_{1}$ is a vibration source, $S_{2}$ is a rectangular plate, which is rigidly baffled and radiates sound into the surrounding fluid. $S_{1}$ and $S_{2}$ are coupled by a piezoelectric actuator, which can be equalized to a spring and an in-parallel force generator. According to the constitutive equations of piezoelectric materials, the axial displacement of a piezoelectric actuator shown in Fig. 2 can be described by Eq. (1).

$$
\Delta L=d_{33} E_{p} L+F / k_{p}
$$

where $\Delta L$ is the axial displacement of the piezoelectric actuator, $\mathrm{d}_{33}$ the piezoelectric strain coefficient for axial displacements in the polarization direction, $\mathrm{L}$ the length of the actuator, $\mathrm{E}_{p}$ the electric field strength, $\mathrm{k}_{p}$ the stiffness 


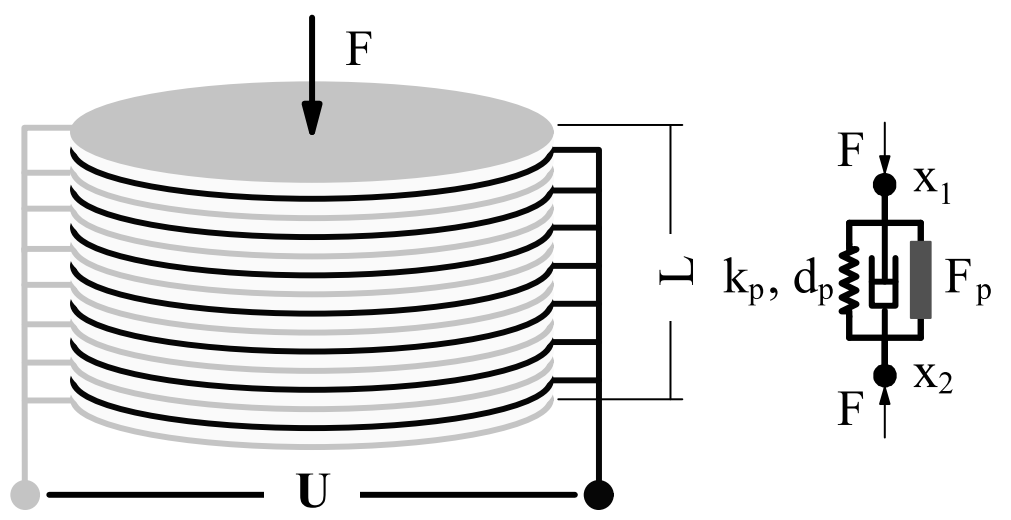

Fig. 2. Piezoelectric actuator.

of the actuator and $\mathrm{F}$ the equivalent concentrated force acting on the stack. At frequencies well below the first resonant frequency of the piezoelectric actuator, Eq. (2) is valid for describing dynamics of the actuator.

$$
k_{p}\left(x_{1}-x_{2}\right)+d_{p}\left(\dot{x}_{1}-\dot{x}_{2}\right)-k_{p} d_{33} n_{p} u=F, k_{p} d_{33} n_{p} u=F_{p}
$$

where $\mathrm{u}$ is the applied voltage as indicated in Fig. $2, \mathrm{n}_{p}$ the number of piezoelectric patches in the actuator.

Suppose $S_{1}$ is a concentrated mass, $S_{2}$ a simply supported plate described by a finite element model and the fluid an ideal medium described by a boundary element model. The vibration equations of $S_{1}$ and $S_{2}$ are given as follows:

$$
\begin{aligned}
& m_{1} \ddot{x}_{1}=F_{1}-F \\
& {\left[\begin{array}{cc}
m_{2} & M_{c} \\
M_{c}^{T} & M_{r}
\end{array}\right]\left\{\begin{array}{l}
\ddot{x}_{2} \\
\ddot{x}_{r}
\end{array}\right\}+\left[\begin{array}{cc}
d_{2} & D_{c} \\
D_{c}^{T} & D_{r}
\end{array}\right]\left\{\begin{array}{l}
\dot{x}_{2} \\
\dot{x}_{r}
\end{array}\right\}+\left[\begin{array}{cc}
k_{2} & K_{c} \\
K_{c}^{T} & K_{r}
\end{array}\right]\left\{\begin{array}{l}
x_{2} \\
x_{r}
\end{array}\right\}=\left\{\begin{array}{c}
F \\
0
\end{array}\right\}-T\{p\}}
\end{aligned}
$$

where $x_{1}$ is the displacement of $\mathrm{S}_{1},\left(x_{2}, x_{r}\right)^{T}$ is the displacement vector of $\mathrm{S}_{2}, x_{1}$ and $x_{2}$ are the displacements of the coupled nodes of $\mathrm{S}_{1}$ and $\mathrm{S}_{2}$, where the piezoelectric actuator is mounted, $x_{r}$ represents the displacements of uncoupled nodes of $\mathrm{S}_{2}, F_{1}$ is the excitation force acting on $\mathrm{S}_{1},\{p\}$ is the pressure acting on the wet surface of $\mathrm{S}_{2}, T$ is the matrix converting fluid pressure to the nodal loads on $\mathrm{S}_{2}$, the matrices on the left-hand side of Eq. (4) are the mass matrix, the damping matrix and the stiffness matrix, respectively. The coupling between $S_{1}$ and $S_{2}$ is given by Eq. (2).

The displacement of $\mathrm{S}_{2}$ can be expressed by the low order vibration modes (in vacuo) to reduce the order of the coupled system, therefore

$$
\left\{\begin{array}{l}
x_{2} \\
x_{r}
\end{array}\right\} \approx \sum_{k=1}^{N} \zeta_{k} \varphi_{k}=\sum_{k=1}^{N} \zeta_{k}\left\{\begin{array}{l}
\varphi_{k}\left(x_{2}\right) \\
\varphi_{k}\left(x_{r}\right)
\end{array}\right\}, \quad \Phi=\left\{\begin{array}{l}
\Phi_{x_{2}} \\
\Phi_{x_{r}}
\end{array}\right\}=\left[\varphi_{1} \varphi_{2} \cdots \varphi_{N}\right]
$$

where $\mathrm{N}$ is the number of retained modes, which is far less than the degrees of freedom of $\mathrm{S}_{2}, \zeta_{k}$ is the kth modal coordinate, $\phi_{k}$ is the kth mode shape, $\Phi$ is the matrix formed by the $\mathrm{N}$ natural modes. In light of Eqs (2)-(5), one can have

$$
\begin{aligned}
& {\left[\begin{array}{cc}
m_{1} & O \\
O & M_{z}
\end{array}\right]\left\{\begin{array}{c}
\ddot{x}_{1} \\
\ddot{\mathrm{Z}}
\end{array}\right\}+\left[\begin{array}{cc}
d_{p} & -\left[d_{p} O\right] \Phi_{x_{2}} \\
-\Phi_{x_{2}}^{T}\left[d_{p} O\right]^{T} & D_{z}
\end{array}\right]\left\{\begin{array}{c}
\dot{x}_{1} \\
\dot{\mathrm{Z}}
\end{array}\right\}+} \\
& {\left[\begin{array}{cc}
k_{p} & -\left[k_{p} O\right] \Phi_{x_{2}} \\
-\Phi_{x_{2}}^{T}\left[k_{p} O\right]^{T} & K_{z}
\end{array}\right]\left\{\begin{array}{c}
x_{1} \\
Z
\end{array}\right\}=\left\{\begin{array}{c}
F_{1}+F_{p} \\
-\Phi_{x_{2}}^{T} F_{p}
\end{array}\right\}-\left\{\begin{array}{c}
O \\
\Phi^{T} T p
\end{array}\right\}}
\end{aligned}
$$

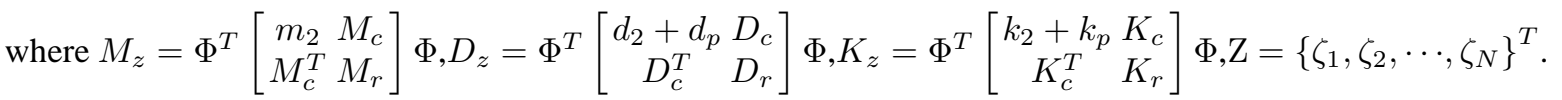

Equation (5) gives the coupled vibration of structures and fluid, in which the pressure $p$ of the fluid is described by the wave equation. 
Suppose $\mathrm{S}_{2}$ is coupled with an infinite body of fluid, the sound pressure $p$ induced by the vibration of $\mathrm{S}_{2}$ is described by the wave equation

$$
\nabla^{2} p=\frac{1}{c^{2}} \frac{\partial^{2} p}{\partial t^{2}} \quad \text { and } \quad \partial p / \partial n=-\rho a(t) \quad \text { on the boundary, }
$$

where $\nabla^{2}$ is the Laplacian operator, $c$ is the sound speed in the fluid, $\rho$ is the fluid density, $n$ is the normal to the wet surface of $\mathrm{S}_{2}, a(t)$ is the acceleration projected to the normal.

To investigate active vibration control involving fluid-structure interaction, Eq. (7) should be simplified so that a reduced lumped time domain model can be derived. Equation (7) will reduce to the Laplace equation if the compressibility of fluid is neglected, i.e. the sound speed c in Eq. (7) is taken as infinity. In this circumstance, the degrees of freedom can be substantially reduced while the accuracy of the coupled model is retained at low frequencies. In fact, the boundary element method can be used to solve the Laplace equation, and the resulting fluid elements are only those on the wet surface of $\mathrm{S}_{2}$. According to the Helmholtz integral equation and the supposition that the sound speed is infinite, the pressure at an arbitrary point within the fluid domain can be given by the integration on the boundary $\Gamma$ (Fig. 1), i.e.

$$
\begin{aligned}
& C(P) p(P)=\int_{\Gamma}\left(\frac{\partial g(Q, P)}{\partial n} p(Q)-g(Q, P) \frac{\partial p(Q)}{\partial n}\right) d \Gamma, \\
& C(P)=\left\{\begin{array}{l}
1, \quad \text { P in fluid } \\
1 / 2, \text { P on } \Gamma \\
0, \quad \text { Otherwise }
\end{array}, \quad g(Q, P)=\frac{1}{4 \pi r(Q, P)}\right.
\end{aligned}
$$

where $p(P)$ is the pressure at $\mathrm{P}, n$ is the normal to $\Gamma, g(Q, P)$ is the Green's function, $r(Q, P)$ is the distance from $\mathrm{Q}$ to $\mathrm{P}$, as shown in Fig. 1. If $\mathrm{P}$ is also located on $\Gamma$, one can obtain from Eq. (7) the matrix relationship between the pressure $\{p\}$ and the acceleration $\left(x_{2}, x_{r}\right)^{T}$ at all nodes on the boundary $\Gamma$ :

$$
H\{p\}=G\left\{\begin{array}{l}
\ddot{x}_{2} \\
\ddot{x}_{r}
\end{array}\right\}=G \Phi\{\ddot{\mathrm{Z}}\}
$$

In light of Eq. (9), Eq. (5) can be rewritten as:

$$
\begin{aligned}
& {\left[\begin{array}{cc}
m_{1} & O \\
O & M_{z}+\Phi^{T} T H^{-1} G \Phi
\end{array}\right]\left\{\begin{array}{c}
\ddot{x}_{1} \\
\ddot{\mathrm{Z}}
\end{array}\right\}+\left[\begin{array}{cc}
d_{p} & -\left[d_{p} O\right] \Phi_{x_{2}} \\
-\Phi_{x_{2}}^{T}\left[d_{p} O\right]^{T} & D_{z}
\end{array}\right]\left\{\begin{array}{c}
\dot{x}_{1} \\
\dot{\mathrm{Z}}
\end{array}\right\}+} \\
& {\left[\begin{array}{cc}
k_{p} & -\left[k_{p} O\right] \Phi_{x_{2}} \\
-\Phi_{x_{2}}^{T}\left[k_{p} O\right]^{T} & K_{z}
\end{array}\right]\left\{\begin{array}{c}
x_{1} \\
Z
\end{array}\right\}=\left\{\begin{array}{l}
F_{1}+F_{p} \\
-\Phi_{x_{2}}^{T} F_{p}
\end{array}\right\}}
\end{aligned}
$$

where the mass matrix has been changed and no pressure load appears on the right-hand side.

In Eq. (10), all the matrices are independent of frequency. The right-hand side of Eq. (10) represents the load vector acting on the coupled system, of which the disturbance force $F_{1}$ excites $\mathrm{S}_{2}$ and thus generates sound in the far field while the control force $F_{p}$ affects the vibration of $\mathrm{S}_{2}$ and further the radiated sound in the far field.

In Eq. (10), $T H^{-1} G$ represents the added fluid mass matrix. For low frequency vibrations, this matrix can reflect accurately the inertial effect of fluid, but for high frequency vibrations, $T H^{-1} G$ overestimates the added inertia of fluid. Therefore, modification should be made in the modeling of the baffled rectangular plate shown in Fig. 1 [6].

First, compute SVD (Singular Value Decomposition) of $T H^{-1} G$, i.e. $T H^{-1} G=U S V^{T}$, where $U, V, S$ are the unitary matrices and the singular value matrix, respectively. Next, find one accurate wet natural frequency of the plate by solving the coupled $\mathrm{FE} / \mathrm{BE}$ model constructed in the frequency domain from the Helmholtz integral equation with $g(Q, P)=\exp (-j \omega r / c) / 4 \pi r$. Then, multiply $S$ with a diagonal matrix $W=\operatorname{diag}\left(\left[1, \cdots, 0.1^{\alpha}\right]\right)$, whose elements are $\left\{\varepsilon(k)^{\alpha} \mid \varepsilon(k)=k \delta, 0 \leqslant k \leqslant N_{s}, N_{s} \delta=1\right\}, N_{s}$ is the number of singular values, $\alpha$ is a constant determined from the wet natural frequency. Finally, give the modified added fluid mass matrix $\tilde{M}=U S W V^{T}$. Therefore, motion equations of the fluid-plate coupled system with modified mass matrix can be given as follows: 


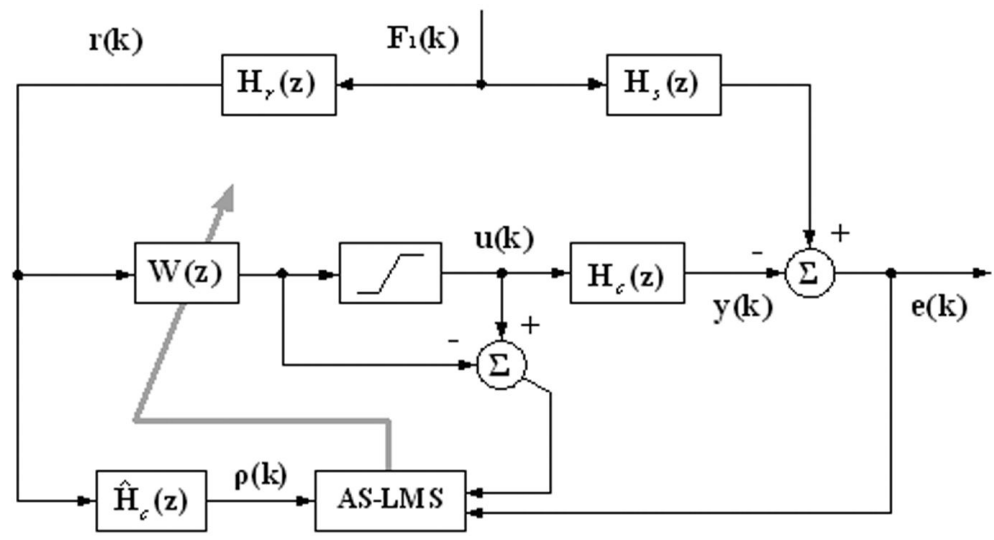

Fig. 3. Adaptive control system.

$$
\begin{gathered}
{\left[\begin{array}{cc}
m_{1} & O \\
O & M_{z}+\Phi^{T} \tilde{M} \Phi
\end{array}\right]\left\{\begin{array}{c}
\ddot{x}_{1} \\
\ddot{\mathrm{Z}}
\end{array}\right\}+\left[\begin{array}{cc}
d_{p} & -\left[d_{p} O\right] \Phi_{x_{2}} \\
-\Phi_{x_{2}}^{T}\left[d_{p} O\right]^{T} & D_{z}
\end{array}\right]\left\{\begin{array}{c}
\dot{x}_{1} \\
\dot{\mathrm{Z}}
\end{array}\right\}+} \\
{\left[\begin{array}{cc}
k_{p} & -\left[k_{p} O\right] \Phi_{x_{2}} \\
-\Phi_{x_{2}}^{T}\left[k_{p} O\right]^{T} & K_{z}
\end{array}\right]\left\{\begin{array}{c}
x_{1} \\
Z
\end{array}\right\}=\left\{\begin{array}{l}
F_{1}+F_{p} \\
-\Phi_{x_{2}}^{T} F_{p}
\end{array}\right\}-\left\{\begin{array}{c}
O \\
\Phi^{T} T p
\end{array}\right\}}
\end{gathered}
$$

To compute the induced sound pressure in the far field, the sound speed c should be a finite value. For the baffled plate in Fig. 1, the instantaneous pressure at a far field point $p(x, y, z)$ can be given by the Rayleigh integration:

$$
p(x, y, z, t)=\frac{\rho}{2 \pi} \int_{\Gamma} \frac{a\left(x_{0}, y_{0}, t-r / c\right)}{r} d \Gamma, r=\sqrt{\left(x-x_{0}\right)^{2}+\left(y-y_{0}\right)^{2}+z^{2}}
$$

where $\left(x_{0}, y_{0}, 0\right)$ is an arbitrary point located on the plate, $(x, y, z)$ is a point located in the semi-infinite space, as shown in Fig. 1, $a\left(x_{0}, y_{0}, t\right)$ is the acceleration of $\left(x_{0}, y_{0}, 0\right)$ at the time $\mathrm{t}, \Gamma$ stands for the surface of the plate.

\subsection{Adaptive control}

In this section, the method of adaptive cancellation is introduced. In fact, adaptive methods have been studied and applied widely [25-27]. According to the theory of linear systems, the discrete form of Eq. (11) and the response can be expressed by

$$
\begin{aligned}
& \phi\left(x_{1}\left(t_{k}\right), \dot{x}_{1}\left(t_{k}\right), x_{1}\left(t_{k+1}\right), \dot{x}_{1}\left(t_{k+1}\right), Z\left(t_{k}\right), \dot{\mathrm{Z}}\left(t_{k}\right), Z\left(t_{k+1}\right), \dot{\mathrm{Z}}\left(t_{k+1}\right)\right)=B_{1} F_{1}\left(t_{k}\right)+B_{2} F_{p}\left(t_{k}\right) \\
& y\left(t_{k}\right)=\vartheta\left(Z\left(t_{k}\right), \dot{\mathrm{Z}}\left(t_{k}\right), F_{1}\left(t_{k}\right), F_{p}\left(t_{k}\right)\right)
\end{aligned}
$$

where $t_{k}$ is the discrete time, $B_{1}, B_{2}$ are the load matrices. The adaptive algorithm is derived on the basis of Eq. (13). As shown in Fig. 3, the adopted adaptive control system is constructed on a modified Filtered-x LMS method - the Anti-saturation LMS (AS-LMS), which can take into account the output saturation of piezoelectric actuators. The recursive formula for the controller coefficients can be deduced and given as follows [28]:

$$
\mathrm{w}(k+1)=f_{u}(u) \mathrm{w}(k)+\mu \frac{f_{u}(u) e(k)+\left(1-f_{u}(u)\right) u}{\gamma+\|\rho(k)\|^{2}} \rho(k)
$$

where $u=\mathrm{w}^{T}(k) \mathrm{r}(k), \mathrm{r}(k)$ is the reference signal, $e(k)$ is the cancelled acceleration responses, $\mathrm{W}(z)$ is the controller, $\rho(k)$ is the output of $\hat{H}_{c}(z)$ to the input $r(k), \hat{H}_{c}(z)$ is the estimate of $H_{c}(z), H_{c}(z)$ is the transfer function of the control channel (from $F_{p}$ to the plate vibration when $F_{1}=0$, as shown in Fig. 4 ), $H_{s}(z)$ is the transfer function of the disturbance channel (from $F_{1}$ to the plate vibration when $F_{p}=0$, as shown in Fig. 4 ), $\mu$ is a constant, $\gamma>0$, $f_{u}(u)$ is the derivative of a saturation function $f(u)$ and $f(u)=\delta\left(1-e^{-u}\right) /\left(1+e^{-u}\right)$. 


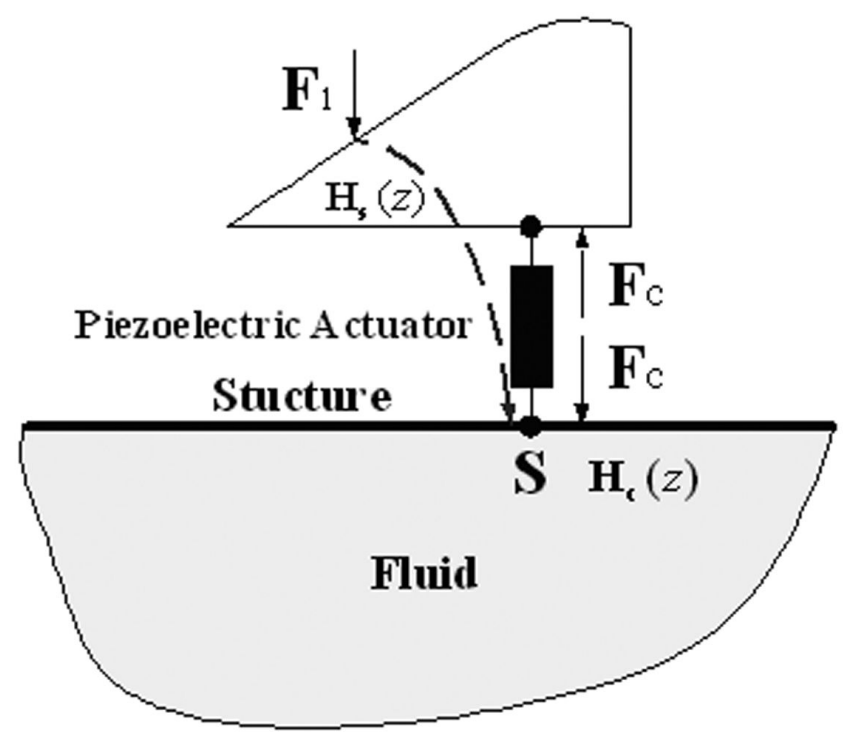

Fig. 4. Vibration isolation with piezoelectric actuators.

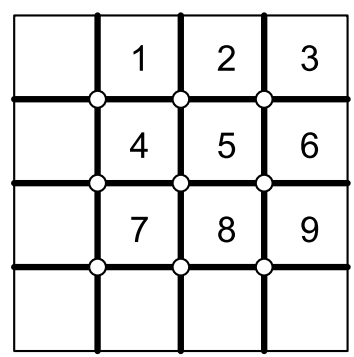

Fig. 5. The plate with cross stiffeners.

\subsection{Sound radiation control}

It is implied from Figs 3 and 4 that sound radiation of the plate is controlled by minimizing the measured vibration of the plate. The drawback of this indirect control is that sound pressure in the far field may not be suppressed as much as the vibration of the plate. According to Eq. (12), sound pressure in the far field can be given approximately by

$$
p(x, y, z, t) \approx \frac{\rho}{2 \pi} \int_{\Gamma} \frac{a\left(x_{0}, y_{0}, t\right)}{r} d \Gamma
$$

This equation implies that minimizing the distributed acceleration may have the same effect as minimizing the far field sound pressure. Hence, in Eq. (14), the control error $e(k)$ can be given as

$$
e(k)=\ddot{x}_{2}(k)+[1, \cdots, 1] \ddot{x}_{r}(k)
$$

where $[1, \cdots, 1] \ddot{x}_{r}(k)$ is the summation of the measured acceleration responses at certain locations of the plate.

\section{Simulation on adaptive control}

Consider a plate with cross stiffeners on one side and connected with a mass through a piezoelectric actuator, which is mounted at the plate center, as shown in Fig 1 and 5. The flat side of the plate is coupled with the fluid. 
Table 1

Dimensions and physical properties

\begin{tabular}{lccccc}
\hline & Dimension (m) & $\begin{array}{c}\text { Density } \\
\left(\mathrm{kg} / \mathrm{m}^{3}\right)\end{array}$ & $\begin{array}{c}\text { Young's } \\
\text { modulus }\left(\mathrm{N} / \mathrm{m}^{2}\right)\end{array}$ & $\begin{array}{c}\text { Poisson's } \\
\text { ratio }\end{array}$ & $\begin{array}{c}\text { Sound } \\
\text { velocity(m/s) }\end{array}$ \\
\hline Plate & $0.8 \times 0.8 \times 0.003$ & 7850 & $2.1 \times 10^{11}$ & 0.3 & - \\
\hline Stiffener & $0.8 \times 0.018 \times 0.003$ & 7850 & $2.1 \times 10^{11}$ & 0.3 & - \\
\hline Fluid & - & 1000 & - & - & 1500 \\
\hline
\end{tabular}

Table 2

Dry and wet natural frequencies

\begin{tabular}{|c|c|c|c|c|c|}
\hline \multirow[t]{2}{*}{ No. } & \multicolumn{2}{|c|}{ Frequencies $(\mathrm{Hz})$} & \multirow[t]{2}{*}{ No. } & \multicolumn{2}{|c|}{ Frequencies $(\mathrm{Hz})$} \\
\hline & FEM(Dry) & $\begin{array}{c}\text { FEM/BEM and } \\
\text { modified(Wet) }\end{array}$ & & FEM(Dry) & $\begin{array}{l}\text { FEM/BEM and } \\
\text { modified(Wet) }\end{array}$ \\
\hline 1 & 61.2 & 17.3 & 4 & 232.5 & 107.8 \\
\hline 2 & 167.4 & 67.9 & 5 & 329.3 & 151.5 \\
\hline 3 & 167.4 & 67.9 & 6 & 329.3 & 160.0 \\
\hline
\end{tabular}

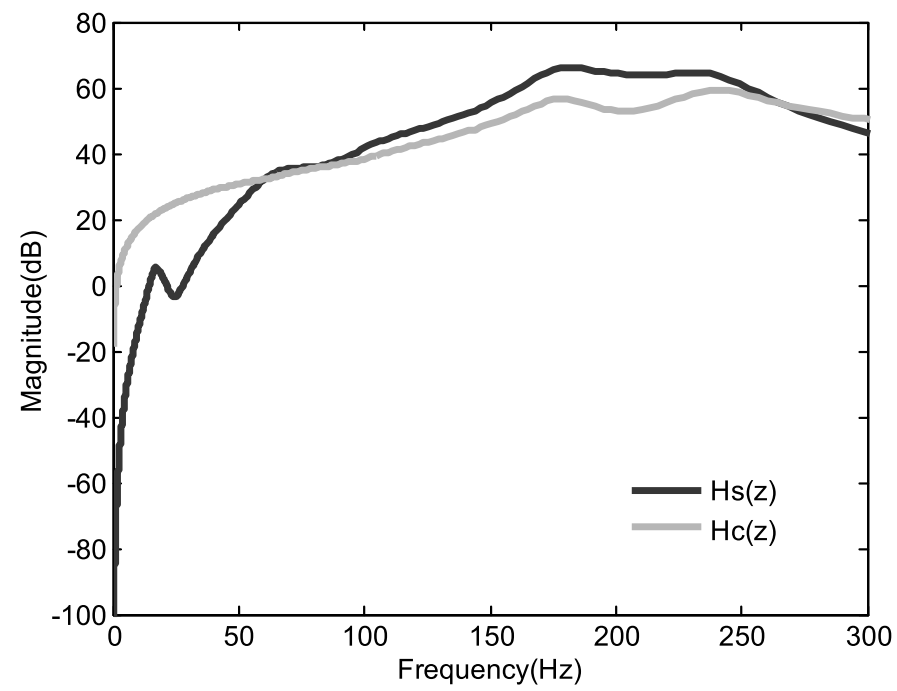

Fig. 6. Frequency response functions.

Dimensions and physical properties of the stiffened plate and the fluid are given in Table 1 . Moreover, $\mathrm{m}_{1}=10 \mathrm{~kg}$, $\mathrm{k}_{p}=3 \times 10^{7} \mathrm{~N} / \mathrm{m}, \mathrm{d}_{p}=250 \mathrm{Ns} / \mathrm{m}$. The plate is modeled with 256 shell elements and the stiffeners are modeled with beam elements. Figure 6 gives the computed frequency response functions corresponding to the disturbance channel and the control channel, respectively. The dry and wet natural frequencies of the plate are listed in Table 2 .

When $\mathrm{m}_{1}$ is excited by a harmonic force, forced vibrations will occur in the stiffened plate. As a result, sound pressure induced by the plate will oscillate at a frequency. In the simulation, no saturation is considered and accordingly $f_{u}(u)=1$. Moreover, adaptive cancellation is carried out to minimize the summed acceleration responses at points 1-9 as shown in Fig. 5. Let $\mathrm{m}_{1}$ be excited by a force of $120 \mathrm{~Hz}$, the acceleration responses and sound pressure with/without adaptive control are simulated and shown in Fig. 7. It can be seen that the summed acceleration and the sound pressure are reduced substantially after control, but the attenuation of sound pressure is related to the location of observation.

\section{Experiment}

The experimental model is a plexiglass water container with a plexiglass plate installed on its top, as shown in Fig. 8. The dimensions of the container are $600 \mathrm{~mm} \times 700 \mathrm{~mm} \times 800 \mathrm{~mm}$ (Height) and the wall thickness is $50 \mathrm{~mm}$. 

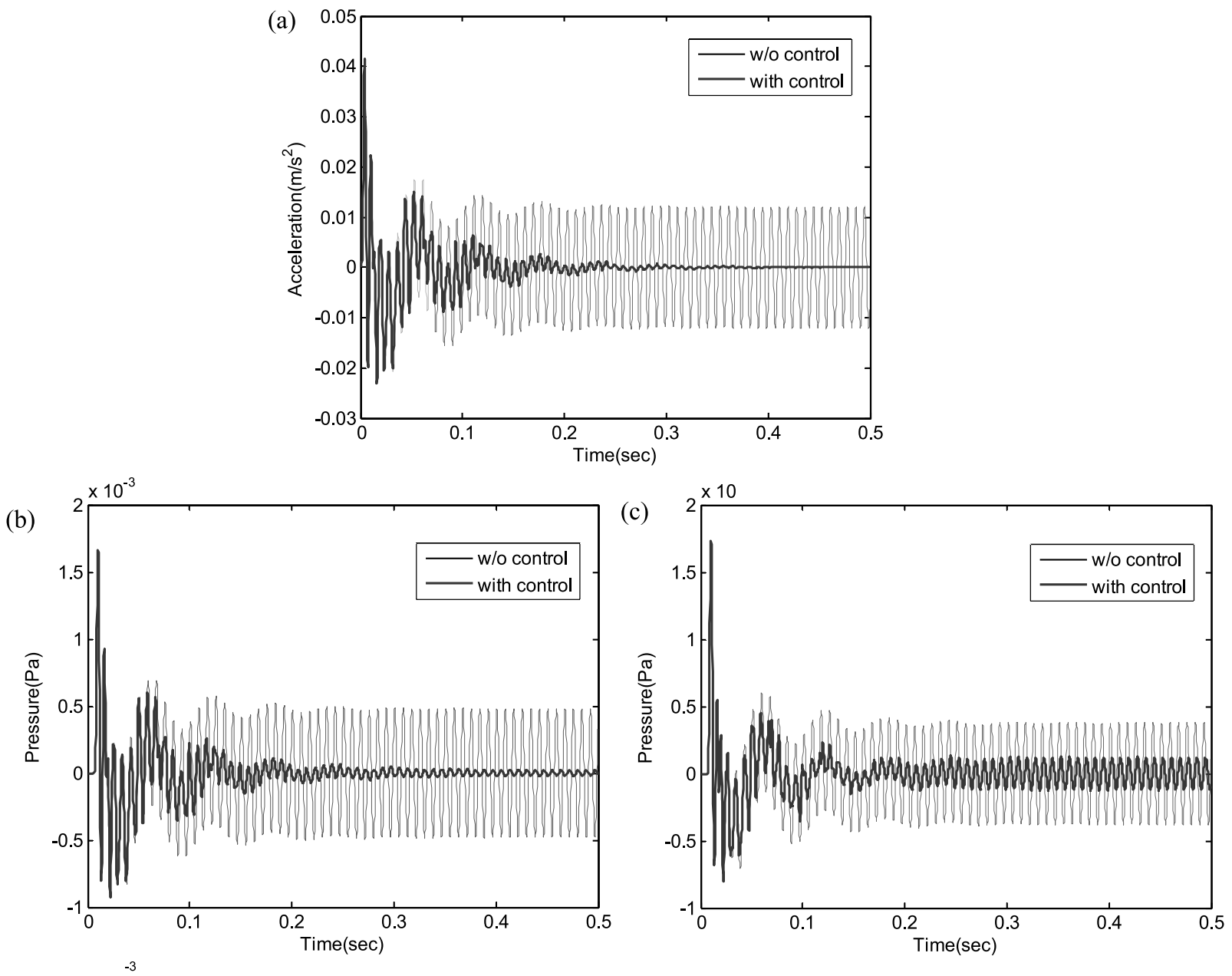

Fig. 7. Acceleration responses and radiated sound pressure $(r=10 / \sqrt{3})$, a) summed acceleration; b) sound pressure at $(0,0,10) \mathrm{m} ; \mathrm{c})$ sound pressure at $(\mathrm{r}, \mathrm{r}, \mathrm{r}) \mathrm{m}$.

The plexiglass plate is $20 \mathrm{~mm}$ thick and the dimensions of the surface in contact with the water are $300 \mathrm{~mm} \times$ $600 \mathrm{~mm}$. In the experiment, the container is nearly full of water so that the lower surface of the plate contacts the water. Four piezoelectric actuators are installed between the plexiglass plate and an aluminium plate of dimensions $300 \mathrm{~mm} \times 180 \mathrm{~mm} \times 8 \mathrm{~mm}$. The fan, having an eccentric mass, is used as a rotary machine and supported on the aluminium plate. Its nominal speed is $2400 \mathrm{rpm}$ and correspondingly the nominal fundamental frequency is $40 \mathrm{~Hz}$. However, the measured frequency oscillates in fact with time, as depicted in Fig. 9. To measure the underwater sound pressure induced by the forced vibration of the plexiglass plate, one sound transducer (Type BK8103) is immersed in the water, $400 \mathrm{~mm}$ below the free water surface. To conduct active cancellation, four accelerometers are installed near the actuators to measure the responses of the plexiglass plate and one accelerometer on the aluminium plate to measure the vibration induced by the fan. Responses of the four accelerometers are summed and the result is used as the control error signal. The controller is a computer with one NI- PCI6259 board inside, which has thirty-two input channels and four output channels. The piezoelectric actuators are PTBS200 of nominal displacement $30 \mu \mathrm{m}$ at $200 \mathrm{~V}$.

FRFs between the excitation of actuators and the sound pressure are measured and depicted in Fig. 10. From this figure, one can find the natural frequencies of the coupled system. The first two peaks are at about $38 \mathrm{~Hz}$ and $82 \mathrm{~Hz}$, corresponding respectively to the first bending mode and the first torsional mode of the plexiglass plate. Figure 11 shows the first two mode shapes, which are measured from the free vibration responses of the selected fifteen points. 


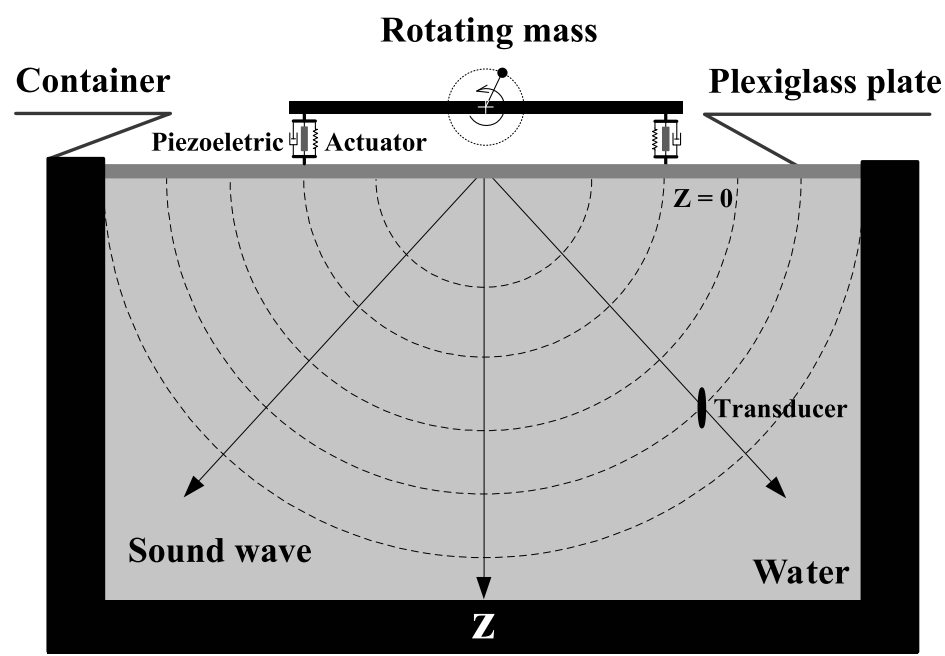

(a)

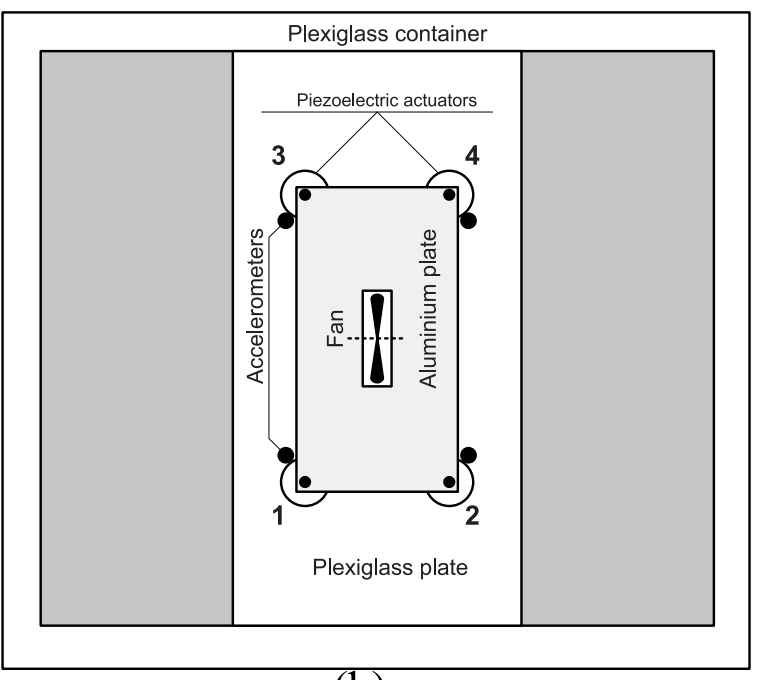

(b)

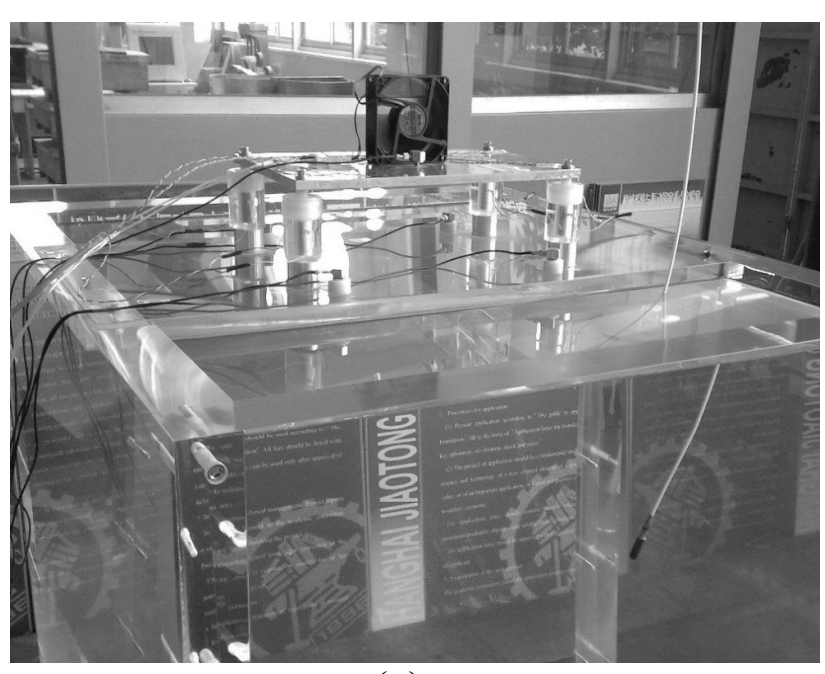

(c)

Fig. 8. Experimental system for active vibration isolation and underwater sound radiation control, a) front view; b) top view; c) photo.

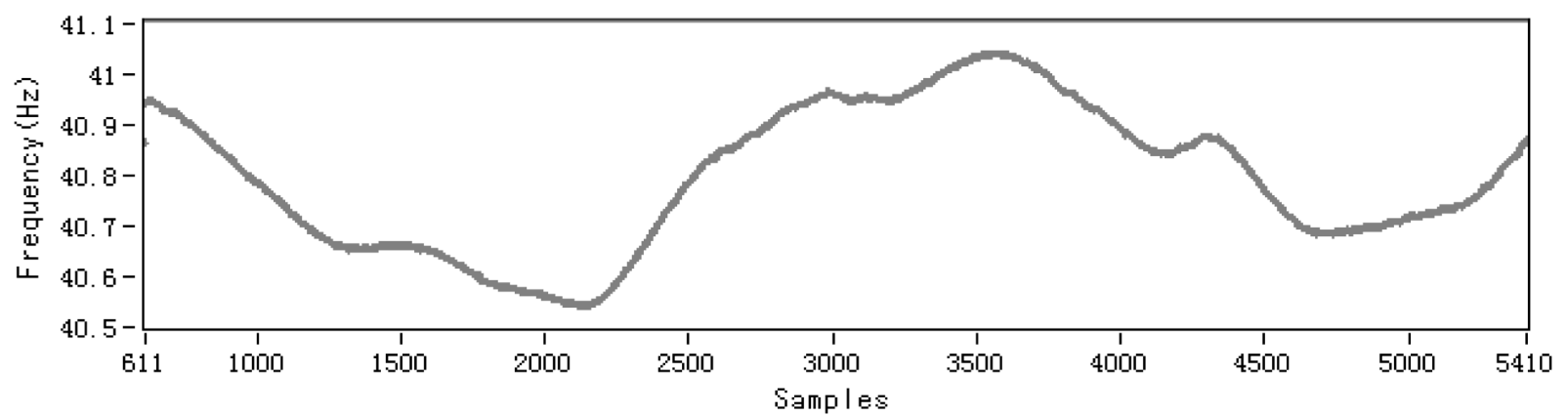

Fig. 9. The measured fundamental frequency of the fan. 


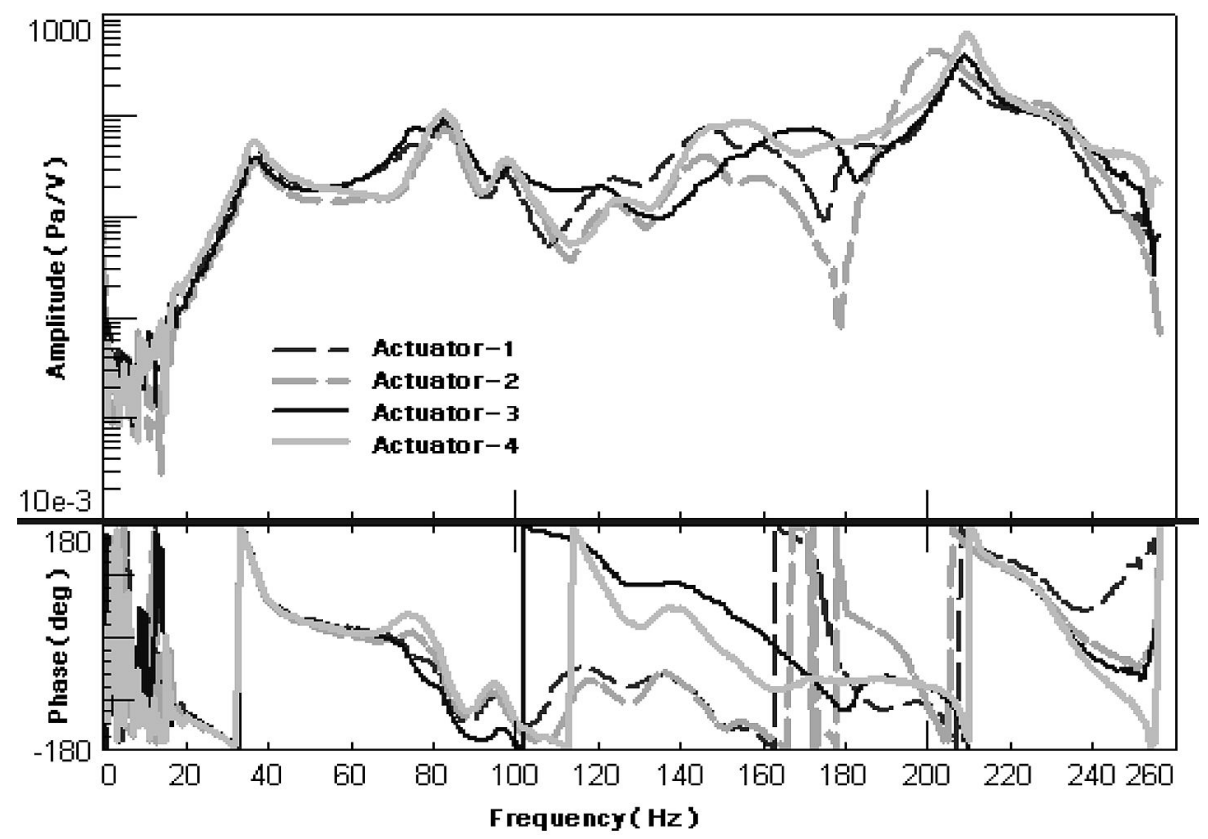

Fig. 10. Measured FRFs between the excitation of the actuators and the sound pressure.

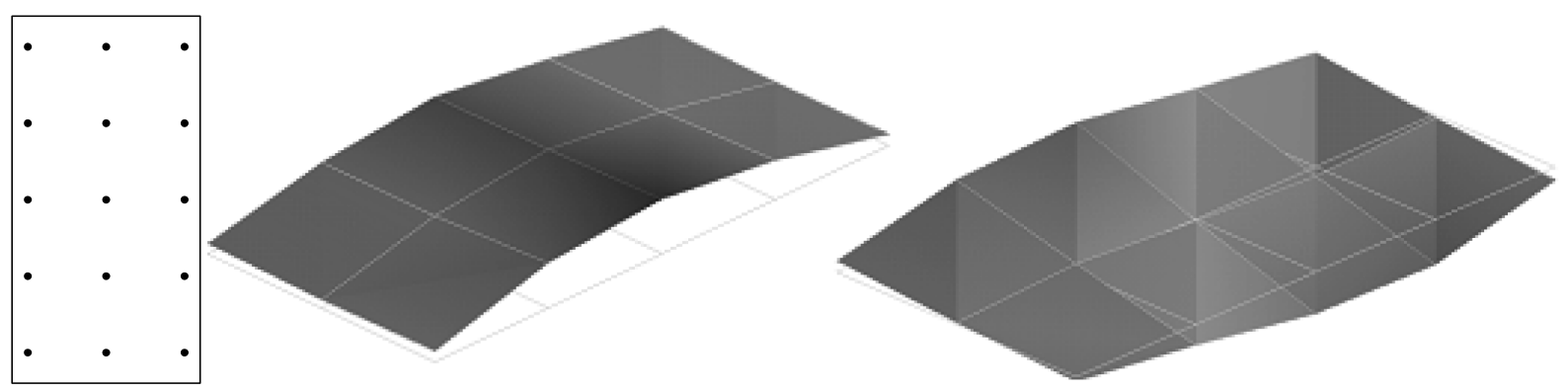

Fig. 11. The first and the second mode shapes of the plate (Left - measurement points; Right - mode shapes.

To demonstrate the effectiveness of adaptive cancellation as well as the piezoelectric actuators in the suppression of vibration and sound, the control action is turned on when the excitation by the fan is in a steady state. The summation of acceleration responses of the four accelerometers and the measured underwater sound pressure in the container is shown in Fig. 12. It can be seen that a noticeable attenuation in vibration and sound occurs at the same time and enters a steady stage after a few seconds of adaptation. Figure 13 gives the spectra of the measured sound pressure before and after control. As can be seen, amplitudes of the first and higher order harmonics are reduced. However, the performance does not reach its optimum as in the simulation due to the limited output force of the actuators, which were in fact not fully excited by the power amplifier in the experiment. It is observed that the phenomenon that a reduced vibration level corresponds to a reduced sound pressure also exists in the experiment albeit the sound field in the bounded water is different from a semi-infinite sound field.

\section{Conclusions}

Active control of vibration and the induced underwater sound radiation is investigated by simulation and experiment. FEM/BEM is applied to model the interaction between the fluid and structures and to establish the coupled 

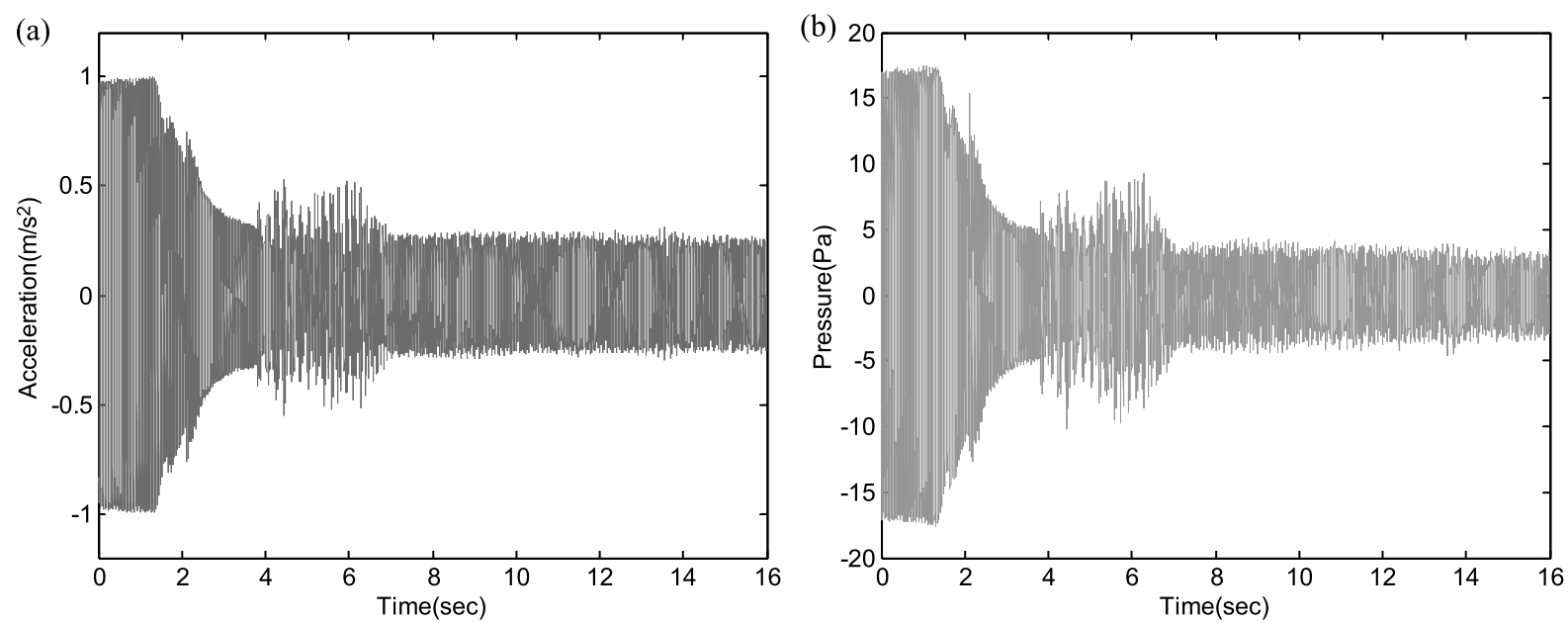

Fig. 12. Measured responses (control action is turned on at $1.4 \mathrm{~s}$ ), a) summed acceleration; b) sound pressure.

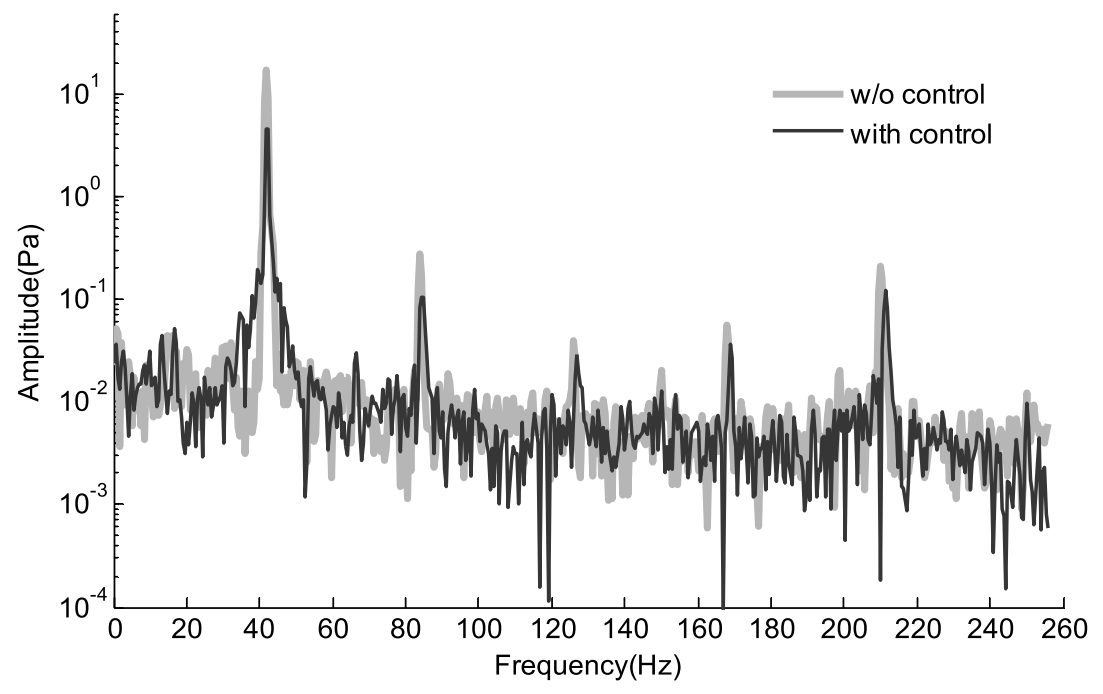

Fig. 13. Sound spectra before and after control.

motion equations. In the modeling, a simplified physical model of the piezoelectric actuator is incorporated into the system and modal truncation is applied to derive a mathematical model of sufficiently small number of degrees of freedom. This model is appropriate for investigating the problems concerning vibration and sound control in the time domain due to its flexibility in dealing with nonlinearity. Simulation is conducted for a system that comprises mainly a piezoelectric actuator and a baffled stiffened plate in the semi-infinite space, and in the experiment, four piezoelectric actuators are used to adaptively cancel summed vibration of the positions where the actuators are mounted. The results have shown that suppression of summed vibration can result in a reduced sound radiation, moreover, the summed vibration as well as the induced underwater sound radiation is noticeably reduced after control action.

\section{Acknowledgment}

This work was supported in part by the NSF of China (Grant No. 10672099, 10972137) and the Laboratory of MSV (Grant No. MSV-MS-2010-10). 


\section{References}

[1] H. Nelisse, O. Beslin and J. Nicolas, A generalized approach for the acoustic radiation from a baffled or unbaffled plate with arbitrary boundary conditions, immersed in a light or heavy fluid, Journal of Sound and Vibration 211 (1998), 207-225.

[2] C.G. Everstine and F.M. Henderson, Coupled finite element/boundary element approach for fluid-structure interaction, Journal of Acoustical Society of America 87 (1990), 1938-1946.

[3] G.Y. Yu, Symmetric collocation BEM/FEM coupling procedure for 2-D dynamic structural-acoustic interaction problems, Computational Mechanics 29 (2002), 191-198.

[4] Z. Tong, Y. Zhang, Z. Zhang and H. Hua, Dynamic behavior and sound transmission analysis of a fluid-structure coupled system using the direct-BEM/FEM, Journal of Sound and Vibration 299 (2007), 645-655.

[5] A. Ergin, The response behaviour of a submerged cylindrical shell using the doubly asymptotic approximation method (DAA), Computers \& Structures 62 (1997), 1025-1034.

[6] Z. Zhang, Y. Chen, X. Yin and H. Hua, Active vibration isolation and underwater sound radiation control, Journal of Sound and Vibration 318 (2008), 725-736.

[7] V.V. Varadan, Z. Wu, S.Y. Hong and V.K. Varadan, Active control of sound radiation from a vibrating structure, Ultrasonics Symposium (1991), 991-994

[8] J.P. Maillard and C.R. Fuller, Active control of sound radiation from cylinders with piezoelectric actuators and structural acoustic sensing, Journal of Sound and Vibration 222 (1999), 363-388.

[9] J.P. Carneal and C.R. Fuller, An analytical and experimental investigation of active structural acoustic control of noise transmission through double panel systems, Journal of Sound and Vibration 272 (2004), 749-771.

[10] H.K. Lee and Y.S. Park, A near-field approach to active control of sound radiation from a fluid-loaded rectangular plate, Journal of Sound and Vibration 196 (1996), 579-593.

[11] C.C. Cheng and J.K. Wang, Structural acoustic response reduction of a fluid-loaded beam using unequally spaced concentrated masses, Applied Acoustics 54 (1998), 291-303.

[12] S. Li and D. Zhao, Numerical simulation of active control of structural vibration and acoustic radiation of a fluid-loaded laminated plate, Journal of Sound and Vibration 272 (2004), 109-124.

[13] S. Daley, J. Hatonen and D.H. Owens, Active vibration isolation in a smart spring mount using a repetitive control approach, Control Engineering Practice 14 (2006), 991-997.

[14] Y. Zhang, A.G. Alleyne and D. Zheng, A hybrid control strategy for active vibration isolation with electrohydraulic actuators, Control Engineering Practice 13 (2005), 279-289.

[15] S. Moona, C. Limb, B. Kima and Y. Park, Structural vibration control using linear magnetostrictive actuators, Journal of Sound and Vibration 302 (2007), 875-891.

[16] Y. Liu, K. Chang and W. Li, Model reference adaptive control for a piezo-positioning system, Precision Engineering 34 (2010), $62-69$.

[17] S. Heamawatanachai and E. Bamberg, Design and characterization of a PZT driven micromachining tool based on single-point tool tip geometry, Precision Engineering (33) (2009), 387-394.

[18] A. Rashid and C.M. Nicolescu, Active vibration control in palletised workholding system for milling International, Journal of Machine Tools \& Manufacture 46 (2006), 1626-1636.

[19] K.Y. Kim, K.H. Park, H.C. Park et al., Performance evaluation of lightweight piezo-composite actuators, Sensors and Actuators A 120 (2005), 123-129.

[20] N. Mechbal, M. Verge, G. Coffignal and M. Ganapathi, Application of a combined active control and fault detection scheme to an active composite flexible structure, Mechatronics 16 (2006), 193-208.

[21] O.M. Fein, L. Gaul and U. Stobener, Vibration Reduction of A Fluid-Loaded Plate By Modal Control, Journal of Intelligent Material Systems and Structures 16 (2005), 541-552.

[22] L. Liu, Z. Zhang and H. Hua, Dynamic characteristics of rotating cantilever plates with active constrained damping layer treatment, Smart Materials and Structures 16 (2007), 1849-1856.

[23] D. Mayer, H. Atzrodt, S. Herold and M. Thomaier, An approach for the model based monitoring of piezoelectric actuators, Computers and Structures 86 (2008), 314-321.

[24] Y. Tian, B. Shirinzadeh, D. Zhang and G. Alici, Development and dynamic modelling of a flexure-based Scott- Russell mechanism for nano-manipulation, Mechanical Systems and Signal Processing 23 (2009), 957-978.

[25] S.J. Elliott, I.M. Stothers and P.A. Nelson, A multiple error LMS algorithm and its application to the active control of sound and vibration, IEEE Transactions on Acoustics, Speech and Signal Processing 35 (1987), 1423-1434.

[26] S. Kim and Y. Park, Active control of multi-tonal noise with reference generator based on on-line frequency estimation, Journal of Sound and vibration 227 (1999), 647-666.

[27] B. Mazeaud and M.A. Galland, A multi-channel feedback algorithm for the development of active liners to reduce noise in flow duct applications, Mechanical Systems and Signal Processing 21 (2007), 2880-2899.

[28] Z. Zhang, F. Hu and J. Wang, On saturation suppression in adaptive vibration control, Journal of Sound and Vibration 329 (2010), 1209-1214. 

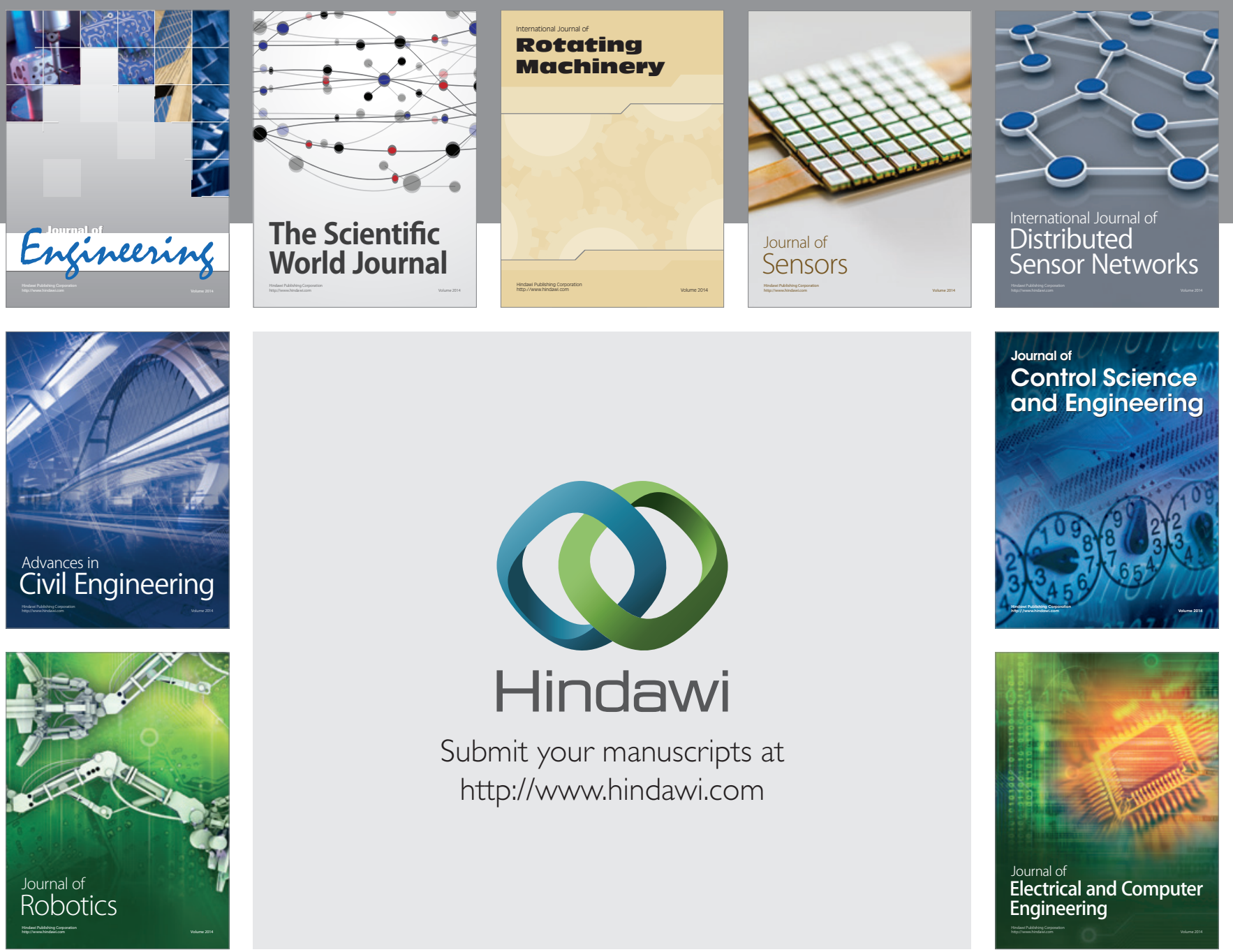

Submit your manuscripts at

http://www.hindawi.com
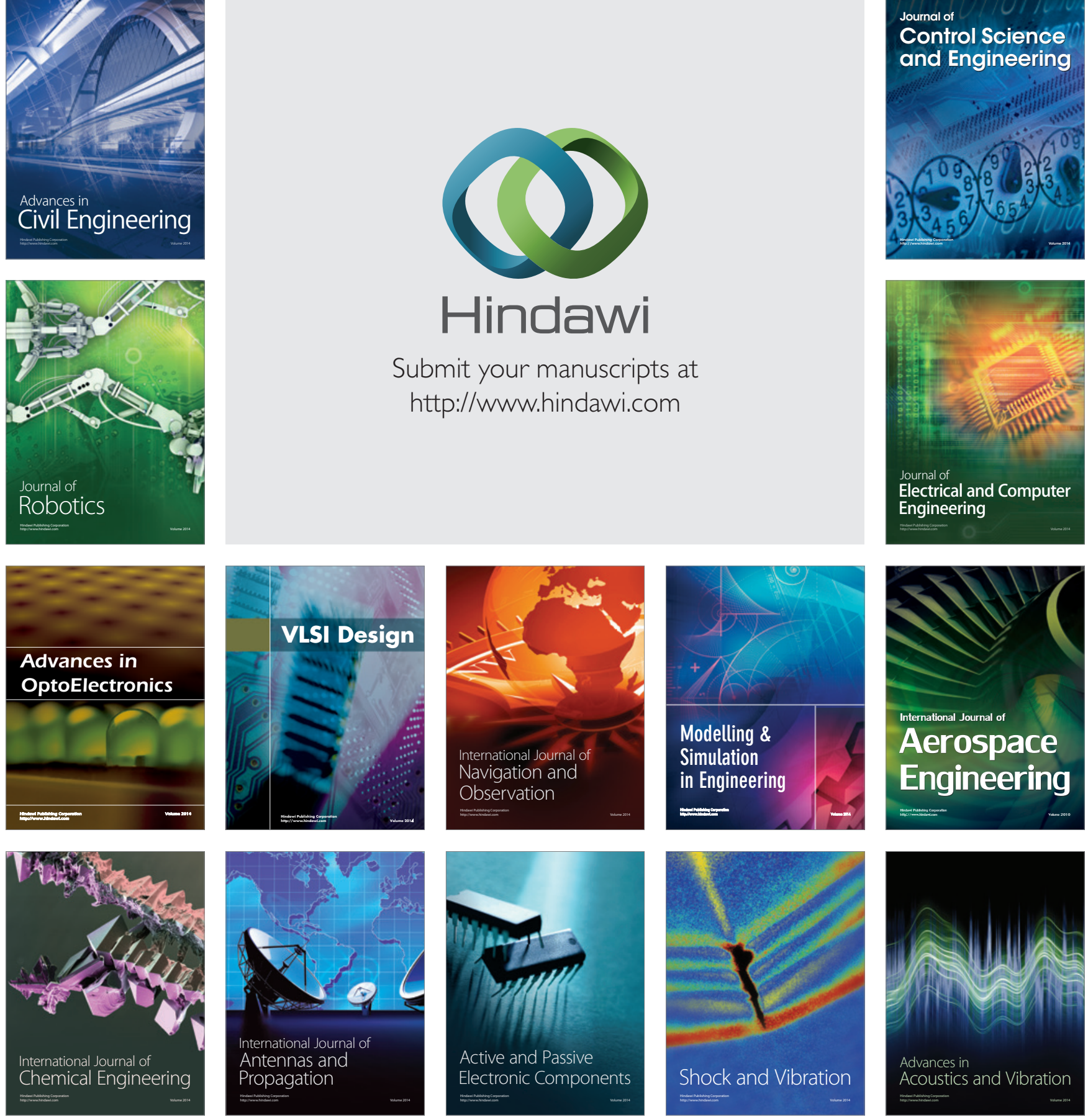